\title{
Cluster randomized trial assessing the effects of rapid ethical assessment on informed consent comprehension in a low-resource setting
}

Adamu Addissie ${ }^{1,2^{*}}$, Serebe Abay ${ }^{3}$, Yeweyenhareg Feleke ${ }^{2}$, Melanie Newport ${ }^{1}$, Bobbie Farsides $^{1}$ and Gail Davey ${ }^{1}$

\begin{abstract}
Background: Maximizing comprehension is a major challenge for informed consent processes in low-literacy and resource-limited settings. Application of rapid qualitative assessments to improve the informed consent process is increasingly considered useful. This study assessed the effects of Rapid Ethical Assessment (REA) on comprehension, retention and quality of the informed consent process.

Methods: A cluster randomized trial was conducted among participants of HPV sero-prevalence study in two districts of Northern Ethiopia, in 2013. A total of 300 study participants, 150 in the intervention and 150 in the control group, were included in the study. For the intervention group, the informed consent process was designed with further revisions based on REA findings. Informed consent comprehension levels and quality of the consent process were measured using the Modular Informed Consent Comprehension Assessment (MICCA) and Quality of Informed Consent (QuIC) process assessment tools, respectively.

Result: Study recruitment rates were $88.7 \%$ and $80.7 \%(p=0.05)$, while study retention rates were $85.7 \%$ and $70.3 \%(p<0.005)$ for the intervention and control groups respectively. Overall, the mean informed consent comprehension scores for the intervention and control groups were $73.1 \%$ and $45.2 \%$, respectively, with a mean difference in comprehension score of $27.9 \%$ (95 \% Cl $24.0 \%-33.4 \% ; p<0.001$,). Mean scores for quality of informed consent for the intervention and control groups were $89.1 \%$ and $78.5 \%$, respectively, with a mean difference of $10.5 \%$ (95 \% Cl $6.8-14.2 \% ; p<0.001)$.

Conclusion: Levels of informed consent comprehension, quality of the consent process, study recruitment and retention rates were significantly improved in the intervention group. We recommend REA as a potential modality to improve informed consent comprehension and quality of informed consent process in low resource settings.
\end{abstract}

Keywords: Informed consent process, Informed consent comprehension, Quality of informed consent, Rapid Ethical Assessment (REA), Ethiopia

\footnotetext{
* Correspondence: adamuaddis@yahoo.com

${ }^{1}$ Brighton and Sussex Medical School, Brighton, UK

${ }^{2}$ Addis Ababa University, Addis Ababa, Ethiopia

Full list of author information is available at the end of the article
} 


\section{Background}

Informed consent comprehension is an important determinant of study compliance and retention [1]. Quality in the informed consent process include adequacy of the information provided; understand-ability of the purpose, benefits and risks of the research; distinction between research and clinical care; voluntariness of participation; recall of signing a consent document; and satisfaction with the consent process as well as the levels of recall and understanding by study participants [2]. Important parameters in monitoring the quality of research implementation in addition to levels of study recruitment include levels recall and understanding of the informed consent information by study participants [3-7]. To this effect, informed consent documents may require to be adapted to the local culture and the educational level of the population, which have been shown to strengthen research efforts, through improved recruitment and retention of participants who better understand their roles and responsibilities [8-10].

Rapid assessment techniques such as Rapid Ethical Assessment (REA) have been documented to play an important role in improving informed consent process for medical research in developing countries by providing better understanding of research contexts [11-14]. REA is a brief (an average of 6 weeks long) qualitative intervention designed to map the ethical terrain of the research setting prior to a research team starts recruiting participants with the purpose of connecting ethical principles to contexts and realities on the ground. REA attempts to discover, describe and respond to the ethical issues specific to a particular research setting, and help researchers to address the issues that genuinely matter to proposed study participants and their community. REA methodology involves interviews, focused discussion and observation conducted among key stakeholders to inform the design of the particular research project. Its findings are utilised to inform and guide the research consent process; ranging from the conception and development of the consent form, to the way consent is obtained. It has been documented that REA is considered relevant and of utmost use for researchers in low income settings [15]. However, the effects of REA on study participants' level of comprehension, recruitment and retention and quality of informed consent have not been documented.

The current study investigated effects of REA on informed consent comprehension, study compliance and quality of informed consent in a low-income setting. We measured consent information comprehension level, recruitment rate, retention rate and quality-of-informed consent among the participants enrolled into another research: "HPV-Subtype Prevalence Study" [16] in the Tigray Regional State of Northern Ethiopia. The HPV study was identified for the REA intervention due to potential ethical issues anticipated in the study.

\section{Methods \\ Study design}

A cluster randomised controlled trial was conducted among participants of an HPV Sero-prevalence study in Northern Ethiopia, from July 8 to August 23, 2013. The intervention for the trial was conducting REA and subsequent revision of the informed consent's content and processes based on the REA findings. The intervention and control groups were compared for levels of comprehension, recruitment, retention (compliance) and perceived qualities of the consent process two weeks after initial consent. More description on the measurements is given in the data collection procedures section below.

\section{Source and study population}

Pregnant women between 18-45 years attending ANC follow-up in four selected health facilities and who were targeted for the HPV sero-prevalence study were both the source and study population for this comprehension assessment. Those with communication challenge, severe illness, gestational-age greater than 34 weeks, or those who were not willing to return after two weeks for the follow-up interview were excluded.

\section{Sample size determination}

Sample size was determined using the sample size formula for double-population proportion -

$$
\mathrm{n}=\frac{\mathrm{P}_{1}\left(1-\mathrm{P}_{1}\right)+\mathrm{P}_{2}\left(1-\mathrm{P}_{2}\right) \mathrm{Xf}(\alpha, \beta)}{\left(\mathrm{P}_{2}-\mathrm{P}_{1}\right)^{2}}
$$

where ;

$\mathrm{n}:$ number of participants per group

$\mathrm{p}_{1}$ : expected levels of comprehension in the control group

$\mathrm{p}_{2}$ : expected levels of comprehension in the intervention group

$\alpha:$ degree of error

$\beta$ : power of the study

Level of comprehension $\left(\mathrm{P}_{1}\right)$ for the control group was estimated based on an earlier study (hence $P_{1}=0.75$ ) [3] We assumed an improvement in the level of comprehension of $13 \%\left(\mathrm{P}_{2}=0.88\right)$ for the intervention group with $80 \%$ power $(\beta=0.80)$, and $95 \%$ confidence interval $(\alpha=0.05)$ (hence $\mathrm{A}(\alpha, \beta)=7.85$ ). After adjusting for $10 \%$ non-response rate, the total sample size required was 300 participants with a 1:1 ratio between intervention and control participants (i.e. 150 in each group). 


\section{Sampling procedure}

Two woredas (districts) targeted for the HPV sero-survey were selected purposively based on comparability. The selected woredas were randomly assigned using a lottery method to either intervention or control. Health centres within each of the woredas were shortlisted based on: availability of Ante Natal Care (ANC) service provision, laboratory services, and appropriate professionals to collect and handle specimen and flow of adequate number of ANC clients. Two health centres from each woredas (i.e. a total of four health centres), were randomly selected from the shortlisted health centres (Fig. 1). Sample size was proportionally allocated to the health facilities based on reported flow of ANC clients in the previous one year reported by the respective health bureau at the district level.

\section{Data collection procedures, instruments and variables}

REA conducted at the beginning of recruitment identified a number of ethical considerations. Based on these findings revisions were suggested on (a) the consent form and information sheet and (b) the consent process and procedures (Table 1). Accordingly the IRB-approved consent form (Additional file 1) was revised for the intervention group (Additional file 2). The revisions made were: use of additional local terminologies, concrete explanations of study information with examples, and contextual clues based on the REA findings. Consent information was provided by reading the information sheet to participants in both groups. In the intervention group, this was accompanied by additional narratives which were attached to the consent document based on the issues identified by REA. Accordingly participants were asked whether they had any questions after reading each section of the form, and once more after the end of the entire reading. Consent in the control group, was obtained by signing on the consent form, but verbally in the intervention group with data collectors signing as witnesses. Sentences and paragraphs in the revised version of informed consent form were relatively short, and the words chosen were more familiar and local. The major characteristics of the two versions are summarized in Table 1.

Level of comprehension of informed consent, and the quality of the consent process were assessed two weeks after consent had been taken. We used Modular Informed Consent Comprehension Assessment (MICCA) and Brief Investigator Questionnaire (BIQ) [17] to measure informed consent comprehension of participants; and Quality of

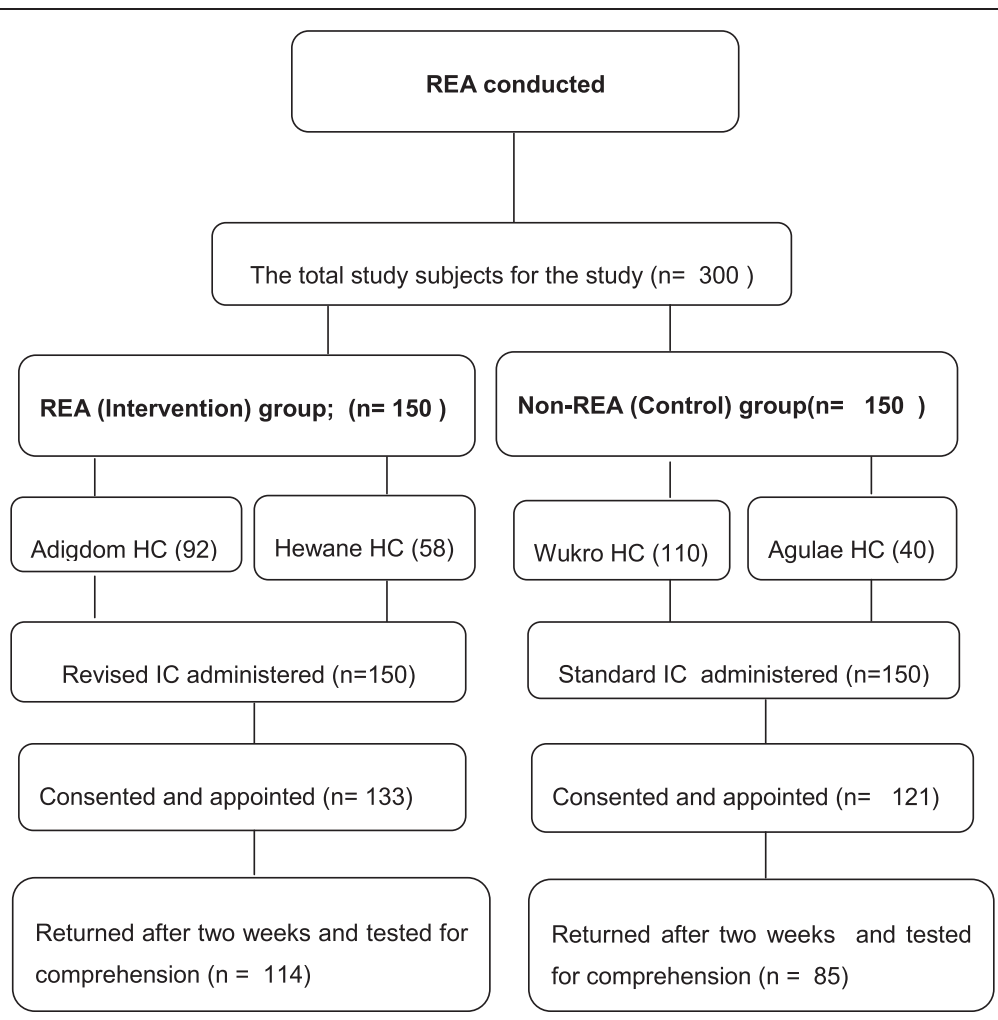

Fig. 1 Flow-Chart showing the sampling procedures for the study. Illustrates the sampling procedures of the study and the steps followed in the recruitment of study subject for the study. Recruitment of the study participants followed the REA (Rapid Ethical Assessment) which was conducted at the very beginning. The study participant were in total 300, with 150 in the intervention and 150 in the control arms. They were selected from two health centres in both instances and the number selected were proportional to the turn-out of patients in each health institution 
Table 1 Comparison of 'standard' and 'revised' versions of information sheet, consent forms and procedures

\begin{tabular}{|c|c|c|}
\hline Consent form information & Revised version & Standard version \\
\hline Font, style & $\begin{array}{l}\text { 12, power geez } \\
\text { uni-code } 1\end{array}$ & $\begin{array}{l}\text { 12, power geez } \\
\text { uni-code } 1\end{array}$ \\
\hline Language & Tigrigna & Tigrigna \\
\hline Average words per sentence & 12.4 & 16.3 \\
\hline Average words per paragraph & 67.1 & 97.8 \\
\hline Average sentences per paragraph & 5.6 & 7.6 \\
\hline Total words & 604 & 587 \\
\hline Total number of paragraphs & 9 & 6 \\
\hline Number of pages & Two and a half & Two \\
\hline Average time taken (minutes) & $\begin{array}{l}\text { Mean }=10 \\
\text { Median }=10\end{array}$ & $\begin{array}{l}\text { Mean }=9.6 \\
\text { Median }=10\end{array}$ \\
\hline Used narrative explanations? & Yes & No \\
\hline $\begin{array}{l}\text { Invited questions on unclear } \\
\text { ideas or concepts }\end{array}$ & $\begin{array}{l}\text { Yes. Written or } \\
\text { included in the } \\
\text { consent form. }\end{array}$ & Yes. Orally \\
\hline Type of consent seeking & Verbal consent & Signed document \\
\hline $\begin{array}{l}\text { Number of days between consent } \\
\text { taking and comprehension } \\
\text { assessment }\end{array}$ & $\begin{array}{l}\text { Mean }=12 \text { days } \\
\text { Median }=12 \text { days }\end{array}$ & $\begin{array}{l}\text { Mean }=11.9 \text { days } \\
\text { Median }=12 \text { days }\end{array}$ \\
\hline
\end{tabular}

Informed Consent (QuIC) [18] to assess quality of the informed consent process. The MICCA and BIQ instruments for our assessment were developed by adopting the existing generic tools (Additional file 3). Both tools have the flexibility to test comprehension specific to a particular trial, yet can be utilized across a variety of trials. MICCA measures comprehension by incorporating both generic and trial-specified approaches. Psychometric evidence suggests that MICCA can be utilized in various trial settings and can produce reliable and valid scores [17].

Comprehension was assessed as a function of understanding and recall. Understanding refers to the concepts explained in the consent process such as purpose, benefits and risk. Recall refers to issues that needed to be memorized such as date of appointment, and specific procedures such as signature, copy of consent given or not [19] (Table 2). A total of 25 questions were used to assess informed consent comprehension of participants; 13 questions were used to assess levels of understanding and 12 questions to asses levels of recall. Test items consisted of both generic and trial specific questions (Additional file 3). Of the 25 test items, 14 were generic test items that appeared on each version of MICCA, 6 were trial-specific test items which were generated based on responses to BIQ and the remaining 5 were trial-specific test items in which the response options for each of the test items are generated based on response to BIQ. QuIC consisted of 13 subjective questions on the perceived quality of the informed consent process (Additional
Table 2 Operational Definitions

Operational definitions

Comprehension Level: the level of participant understanding and recall of the information they were given through the consent document. Assessment is based on the Comprehension Test administered two weeks into the study. Comprehension was categorized into three levels [high :75\% or greater; medium: between $50 \%$ and $75 \%$; low: $50 \%$ or less ] adapted from earlier studies [3]. Its components included Recall and Understanding. Recall: success in selecting or remembering the correct statement Understanding: correctness of interpretations of statements presented Study Recruitment rate: Recruitment rate is the proportion (percentage) of participants who voluntarily decide to participate in the study out of those who were approached to take part. Study Compliance (or Retention) rate: Measures compliance to follow-up and appointments as directed in the visit before. Compliance rate refers to the proportion (percentage) of participant that attended their appointment at the specified time divided by the number of participants who were given an appointment and agreed to come. Quality of informed consent process assessment: a measure of the quality of consent process concerning information adequacy, participant perception and satisfaction of the process, and the situation of decision making during the recruitment process. Scoring levels were the same as for comprehension.

file 3). Each correct response was scored as 1, while each incorrect response was scored 0. For multiple choices with more than one correct response a score value of 1 was given for each possible answer. All the correct answers for understanding and recall were separately summed and calculated out of hundred.

MICCA, BIQ and QuIC-based questionnaires for the study were initially prepared in English (see Additional file 3) and then translated to Tigrigna, the local language. The Tigrigna version was translated back to English to check for consistency of meaning. Data collectors who recruited participants and obtained initial consent were nurses from the respective health centres, who had been trained for the study. Comprehension assessment was done by data collectors who had been trained on the use of the assessment tools. Data collectors were supervised by public health experts with postgraduate qualifications.

\section{Data analysis}

Data were pre-coded and entered into Epi-Info version 3.5.1. Data were cleaned and edited using simple frequencies and cross tabulation and exported to SPSS Version 16 for further analysis. Descriptive statistics were generated for describing the study population in relation to relevant socio-demographic variables. Regression analysis were done for comparative analysis and assessing association between the major outcome variables and associated factors such as educational status, occupation and previous participation in research. Risk ratios with $95 \%$ confidence interval were used to determine levels of significance. Multivariate logistic regression model was used to control for confounders and adjust the measures of association. 


\section{Results}

\section{REA findings}

Rapid Ethical Assessment conducted at the beginning of recruitment identified a number of ethical considerations, based on which the consent form and process for the intervention group were revised. Major REA findings included low awareness of the concept of research (therapeutic misconception), presence of indigenous descriptions and terminologies for technical disease-related terms; concerns about biological samples; major discomfort around signing a consent form; discomfort with male enumerators; suspicions around the study selection criteria; concerns with outsiders coming to the community with incentives; and concerns over test results and their implications. These findings dictated the need for further elaboration on some of the components of the research, for extended discussion on the issues identified, and for changes in the consenting mechanism.

\section{Socio-demographic and economic characteristics of study participants}

A total of 300 pregnant women were approached for the study, each half from control and intervention locations. Of the 254 who consented (133 from intervention and 121 from control sites), 199 (114 from intervention and 85 from control site) also came to the follow-up visit after two weeks and participated in the comprehension assessment.

The mean age $( \pm 2 \mathrm{SD})$ of the participants who complied with the follow-up appointment was $27.3(+/-6.3)$ years. Majority (83.4\%) were married; from the Tigre ethnic group (99.5\%); Orthodox by religion (86.4\%); able to read and write (70.8\%); and had a monthly family income of $>1000$ Ethiopian Birr ETB) $(61.8 \%)$ which is equivalent to 50 USD. For most participants (72.9\%), it was the first time they had participated in any medical research (Table 3).

\section{Recruitment and retention rates}

The overall study recruitment rate was $84.7 \%$ with $88.7 \%$ in the intervention and $80.7 \%$ in the control groups $(p=0.05)$. The overall study retention rate was $78.3 \%$ with $85.7 \%$ for the intervention and $70.3 \%$ for the control groups $(p<0.005)$. The loss-to-follow-up rates after initial consent were $14.3 \%$ and $29.8 \%$ in the intervention and control groups respectively.

\section{Informed-consent comprehension scores}

In the control group, majority (77.6 \%) had low comprehension score (50 \% or less). Only $4.7 \%$ had high comprehension ( $75 \%$ or greater) score. The remaining $17.6 \%$ had medium comprehension scores (between $50 \%$ and $75 \%$ ). In the intervention group, $43 \%$ of participants had high comprehension scores (75 \% or greater), only $8.8 \%$ had a low comprehension score (50 \% or less) and the remaining $48.2 \%$ had a medium score (between $50 \%$ and $75 \%$ ). Participants in the intervention group were 4.6 times more likely to have medium comprehension scores and were 14.5 times more likely to have high comprehension scores than participants in the control group [Table 4]. The mean comprehension scores of the two groups (intervention and control) for all the 25 questions used in the comprehension test are presented in Table 5.

Participants in the intervention group obtained an average score of $73.1 \%$ in overall comprehension; $73.2 \%$ in the recall and $73.0 \%$ in the understanding sections. Participants in the control group obtained an average score of $45.2 \%$ in the overall comprehension; $44.8 \%$ in the recall and $45.6 \%$ in the understanding categories. There were statistically significant mean differences in all the three categories between the intervention and control groups $(p<0.001)$. There was a significant net difference in the mean score comprehension in almost all of the components in the intervention group compared to the standard. The highest mean difference $(42.7 \%)$ was observed in the understanding of participant rights. There was no statistically significant mean score difference in disease-related information between the intervention and control groups [Table 6].

Further analysis was done for potential socio-demographic factors associated with comprehension irrespective of REA. Participants with higher educational level (secondary and above) were found to be about 6.7 times more likely (AOR $=6.68,95 \%$ CI 1.01 to 44.03 ) to obtain high scores $(>=75 \%)$ in the comprehension assessment than those with no formal education. Participants who had ever participated in any medical research were 2.8 times more likely to score high (AOR $=2.77,95 \%$ CI 1.01 to 7.58 ) than those who had never. Occupation and income was also found significantly associated with high score of comprehension [Table 7].

\section{Quality of informed consent}

Overall, the mean informed consent quality assessment score for the control group was $78.5 \%$, and that for the intervention group was $89.1 \%$ [Table 8]. More than eighty percent (81.2 \%) in the control and $99.1 \%$ in the intervention group were satisfied with the overall consent process, while a statistically significant mean difference in the overall mean quality of informed consent scores between the intervention and control groups. Statistically significant difference was detected in all but three components of quality assessment [Table 8].

\section{Discussion}

The study demonstrated the possible impacts of REA on study retention rate, levels of comprehension and quality of the informed consent process among study participants 
Table 3 Demographic and economic characteristics of respondents of WukiroKilte-Awulaelo and Hintal-wajiratworedas (districs), Tigray region, Northern Ethiopia, July 2013

\begin{tabular}{|c|c|c|c|}
\hline Variable $(n=199)$ & Intervention $\mathrm{f}(\%)(n=114)$ & Control $\mathrm{f}(\%)(n=85)$ & Total $f(\%)(n=199)$ \\
\hline \multicolumn{4}{|l|}{ Age in years } \\
\hline $18-24$ & $34(29.8 \%)$ & $38(44.7 \%)$ & $72(36.2)$ \\
\hline $25-31$ & 45 (39.5 \%) & 30 (35.3\%) & $75(37.7)$ \\
\hline $32-38$ & $30(26.3 \%)$ & $14(16.5 \%)$ & $44(22.1)$ \\
\hline $39-45$ & $5(4.4 \%)$ & $3(3.5 \%)$ & $8(4)$ \\
\hline Mean $( \pm S D)$ & $27.9( \pm 6.2)$ & $26.4( \pm 6.2)$ & $27.3( \pm 6.3)$ \\
\hline \multicolumn{4}{|l|}{ Marital status } \\
\hline Married & $99(86.8 \%)$ & $67(78.8 \%)$ & $166(83.4)$ \\
\hline Single & $5(4.4 \%)$ & $8(9.4 \%)$ & $13(6.5)$ \\
\hline Divorced & 7 (6.1 \%) & $6(7.1 \%)$ & $13(6.5)$ \\
\hline Widowed & $3(2.6 \%)$ & $4(4.7 \%)$ & $7(3.5)$ \\
\hline \multicolumn{4}{|l|}{ Educational status } \\
\hline Not able to read and write & $37(32.5 \%)$ & $21(24.7 \%)$ & $58(29.1)$ \\
\hline Able to read and write & $11(9.6 \%)$ & $6(7.1 \%)$ & $17(8.5)$ \\
\hline Primary school & $29(25.4 \%)$ & $21(24.7 \%)$ & $50(25.1)$ \\
\hline Secondary school & $24(21.1 \%)$ & $27(31.8 \%)$ & $51(25.6)$ \\
\hline Diploma and above & $13(11.4 \%)$ & $10(11.8 \%)$ & $23(11.6)$ \\
\hline \multicolumn{4}{|l|}{ Religion } \\
\hline Orthodox Christian & $100(87.7 \%)$ & $72(84.7 \%)$ & $172(86.4)$ \\
\hline Muslim & $13(9.6 \%)$ & $10(11.8 \%)$ & $23(11.6)$ \\
\hline Others (Catholic/Protestant) & 1 (0.9\%) & $3(3.5 \%)$ & $4(2.0)$ \\
\hline \multicolumn{4}{|l|}{ Mother tongue } \\
\hline Tigrigna & $114(100 \%)$ & $83(97.6 \%)$ & $197(99)$ \\
\hline Amharic & 0 & $1(1.2 \%)$ & $1(0.5)$ \\
\hline Others & 0 & $1(1.2)$ & $1(0.5)$ \\
\hline \multicolumn{4}{|l|}{ Occupation } \\
\hline Housewife & $24(21.1 \%)$ & $47(55.3 \%)$ & $71(35.7)$ \\
\hline Farmer & $38(33.3 \%)$ & $4(4.7 \%)$ & $42(21.1)$ \\
\hline Merchant & $28(24.6 \%)$ & $6(7.1 \%)$ & $34(17.1)$ \\
\hline Government employee & $15(13.2 \%)$ & $13(15.3 \%)$ & $28(14.1)$ \\
\hline Private employee & $4(3.5 \%)$ & $10(11.8 \%)$ & $14(7.0)$ \\
\hline Others (student, jobless) & $5(4.4 \%)$ & $5(5.9 \%)$ & $10(5.0)$ \\
\hline \multicolumn{4}{|l|}{ Family monthly income } \\
\hline Less than 500ETB ${ }^{a}$ & $6(5.3 \%)$ & $10(11.8 \%)$ & $16(8.0)$ \\
\hline 500-1000ЕТВ & $26(22.8 \%)$ & $34(40.0 \%)$ & $60(30.2)$ \\
\hline Greater than 1000ETB & $82(71.9 \%)$ & $41(48.2 \%)$ & $123(61.8)$ \\
\hline Participated in medical research previously & $21(18.4 \%)$ & $15(17.6 \%)$ & $36(18.1)$ \\
\hline
\end{tabular}

${ }^{a_{1}}$ USD (US Dollar) $19 \mathrm{ETB}$ and GBP $28.5 \mathrm{ETB}$

in a low-income setting. REA conducted prior to recruitment of participants revealed a number of relevant and context-specific ethical issues concerning the HPV study and its targeted study population. These findings helped in adapting the study information sheet, consent form and consent procedures to the local culture and educational level of the population. The HPV study was selected for the REA intervention due to expressed interest from the investigators and expressed concerns in relation to the sensitivity of the issues as the study deals with cancer of the reproductive organ and sexually transmitted infection. Such parameters which suggested that REA will be relevant 
Table 4 Level of informed consent comprehension in the intervention and control groups, WukiroKilte-Awulaelo and Hintal-wajiratdistrics, Tigray region, Northern Ethiopia, July 2013

\begin{tabular}{lllll}
\hline Comprehension Score & Intervention Group $f(\%)(n=114)$ & Control Group $f(\%)(n=85)$ & RR $(95 \%$ Cl) & $P$ value \\
\hline Low $[<=50 \%]$ & $10(8.8)$ & $66(77.6)$ & 1.00 & $4.6(2.9,7.3)$ \\
Medium $[50 \%-75 \%]$ & $55(48.2)$ & $15(17.6)$ & $14.5(5.6,37.9)$ & $<0.0001$ \\
High $[>=75 \%]$ & $49(43.0)$ & $4(4.7)$ & $<0.0001$
\end{tabular}

Table 5 Mean informed consent comprehension score (CS) of participants, Tigray region, Northern Ethiopia, July 2013

\begin{tabular}{|c|c|c|c|c|c|}
\hline & \multirow[t]{2}{*}{ Consent component } & \multicolumn{2}{|l|}{ Mean \% CS } & \multirow[b]{2}{*}{$\begin{array}{l}\text { Mean difference } \\
(95 \% \mathrm{Cl})\end{array}$} & \multirow[b]{2}{*}{$P$ value } \\
\hline & & $\begin{array}{l}\text { Intervention } \\
(N=114)\end{array}$ & $\begin{array}{l}\text { Control } \\
(N=85)\end{array}$ & & \\
\hline 201 & This health related study is a form of a research ${ }^{\mathrm{a}}$. [True] & 44.7 & 30.6 & $14.1(0.5,27.8)$ & 0.043 \\
\hline 202 & Obligation to participate in this medical research. & 19.3 & 8.2 & $11.1(1.2,21.0)$ & 0.029 \\
\hline 203 & Told who is funding this researcha. [True] & 46.5 & 34.1 & $12.4(1.5,26.2)$ & 0.080 \\
\hline 204 & Told the total number of people that participate in this research ${ }^{\mathrm{a}}$. [False] & 57.9 & 44.7 & $13.2(0.9,27.3)$ & 0.066 \\
\hline 205 & Except study team no one will be allowed to see my health information ${ }^{a}$. [True] & 65.8 & 81.2 & $15.4(27.9,2.9)$ & 0.016 \\
\hline 206 & I will be told test results from this research ${ }^{\mathrm{b}}$. [False] & 76.3 & 15.3 & $61.0(49.7,72.3)$ & $<0.0001$ \\
\hline 207 & Treated for the infection tested by this research ${ }^{b}$. [False] & 63.2 & 11.8 & $51.4(39.4,63.4)$ & $<0.0001$ \\
\hline 208 & I have been told contact person address ${ }^{\mathrm{a}}$. [True] & 71.9 & 41.2 & $30.8(17.5,44.0)$ & $<0.0001$ \\
\hline 209 & I will get a special care in my regular ANC follow up ${ }^{b}$. [False] & 64.0 & 55.3 & $8.7(5.1,22.6)$ & 0.215 \\
\hline 210 & My participation in the study can be stopped at any time ${ }^{a}$. [True] & 81.6 & 20.0 & $61.6(5.0,72.8)$ & $<0.0001$ \\
\hline 211 & I will be asked for costs related to my participation in this study ${ }^{a}$. [False] & 80.7 & 50.6 & $30.1(17.5,42.7)$ & $<0.0001$ \\
\hline 212 & I will be paid or got any incentive for participating in this studya. [False] & 95.6 & 42.4 & $53.3(43.1,63.4)$ & $<0.0001$ \\
\hline 213 & The sample taken in this study can be used for other purpose ${ }^{a}$. [False] & 87.7 & 49.4 & $38.3(26.6,50.0)$ & $<0.0001$ \\
\hline 301 & Selection to participate in this study ${ }^{a}$. $[A]$ & 99.1 & 78.8 & $20.3(12.5,28.1)$ & $<0.0001$ \\
\hline 302 & When to visit a doctor to avoid cervical cancer ${ }^{\mathrm{b}}$. [B] & 67.5 & 51.8 & $15.8(2.1,29.5)$ & 0.024 \\
\hline 303 & Who analyze and discuss test results ${ }^{\mathrm{b}}$. [A] & 95.6 & 51.8 & $43.3(33.6,54.1)$ & $<0.0001$ \\
\hline 304 & At what time can leave the study? ${ }^{\mathrm{a}}[\mathrm{A}]$ & 64.0 & 12.9 & $51.1(39.0,63.1)$ & $<0.0001$ \\
\hline 305 & Agreed or signed to participate in this research mean ${ }^{\mathrm{a}}$. [C] & 87.7 & 61.2 & $26.5(15.1,38.0)$ & $<0.0001$ \\
\hline 306 & Can stop participation after you signed to participate? ${ }^{\mathrm{a}}[\mathrm{A}]$ & 73.3 & 21.2 & $52.5(40.4,64.6)$ & $<0.0001$ \\
\hline 307 & Any difference made to regular ante natal care if not participate $?^{\mathrm{a}}[\mathrm{B}]$ & 85.1 & 81.2 & $3.9(6.6,14.5)$ & 0.466 \\
\hline \multirow[t]{2}{*}{308} & $\begin{array}{l}\text { The main purpose(s) of the study? } \\
\text { To know more about cancer disease in Ethiopia [A] }\end{array}$ & 67.5 & 56.5 & $11.1(2.6,24.7)$ & 0.111 \\
\hline & To introduce vaccine to benefit future generation of girls [B] & 91.2 & 47.1 & $44.2(33.1,55.3)$ & $<0.0001$ \\
\hline 309 & $\begin{array}{l}\text { The main benefit(s) taking part in this research? } \\
\text { Future generation of girls but not me will benefit. [C] }\end{array}$ & 69.3 & 36.5 & $32.8(19.5,46.2)$ & $<0.0001$ \\
\hline \multirow[t]{2}{*}{310} & $\begin{array}{l}\text { Procedure(s) asked to take part in? } \\
\text { Giving a small amount blood for test }[\mathrm{A}]\end{array}$ & 96.5 & 65.9 & $30.6(21.0,40.3)$ & $<0.0001$ \\
\hline & Giving vaginal secretion for test $[C]$ & 91.2 & 56.5 & $34.8(23.7,45.8)$ & $<0.0001$ \\
\hline 311 & $\begin{array}{l}\text { Task(s) asked to complete? } \\
\text { Attend appointment }[\mathrm{A}]\end{array}$ & 98.2 & 83.5 & $14.7(7.3,22.2)$ & $<0.0001$ \\
\hline \multirow[t]{2}{*}{312} & $\begin{array}{l}\text { Side effect(s) that might occur during blood drawing for test? } \\
\text { Pain or bruising on the vein }[\mathrm{A}]\end{array}$ & 57.0 & 41.2 & $15.8(1.8,29.9)$ & 0.027 \\
\hline & Bleeding at the site of the needle [B] & 47.4 & 34.1 & $13.3(0.6,27.1)$ & 0.061 \\
\hline \multicolumn{2}{|c|}{ Over all C-Score } & 73.1 & 45.2 & $27.9(23.96,31.87)$ & $<0.0001$ \\
\hline
\end{tabular}

\footnotetext{
a Generic test item- These test items appear on each version of MICCA
}

${ }^{b}$ Trial specific test item- These test items do not appear on every version of the MICCA. They are generated based on responses to BIQ

'Trial specific test items appear on each version of the MICCA. The response option for each of these test items are generated based on response to BIQ 
Table 6 Comparison of percentage of participants giving correct responses to the main consent components in the intervention and standard group, WukiroKilte-Awulaelo and Hintal-wajiratworedas(districts), Tigray region, Northern Ethiopia, July 2013

\begin{tabular}{|c|c|c|c|c|}
\hline Consent component & $\begin{array}{l}\text { Mean \% CS Intervention } \\
N=114)\end{array}$ & $\begin{array}{l}\text { Mean \% CS Control } \\
(N=85)\end{array}$ & $\begin{array}{l}\text { Mean difference } \\
(95 \% \mathrm{Cl})\end{array}$ & $P$ \\
\hline Disease information [Q302] & 67.54 & 51.77 & $15.8(2.1,29.5)$ & 2.4 \\
\hline Information about the study[Q201, 203, 204, 208] & 55.26 & 37.64 & $17.6(9.46,25.76)$ & $<0.001$ \\
\hline Aim /purpose of the study[Q308A, 308B] & 79.38 & 51.76 & $27.62(19.53,35.70)$ & $<0.001$ \\
\hline Selection criteria [Q301] & 99.12 & 78.82 & $20.30(12.50,28.10)$ & $<0.001$ \\
\hline Procedure of sample collection [Q310A, 310C] & 93.86 & 61.18 & $32.68(25.21,40.86)$ & $<0.001$ \\
\hline Tasks to be performed by participants [Q311A] & 98.25 & 83.53 & $14.70(7.30,22.20)$ & $<0.001$ \\
\hline Risk of the study/ side effect of sample collection [Q312A, 312B] & 52.19 & 37.65 & $14.55(4.05,25.04)$ & 0.007 \\
\hline Benefit of the study [Q206, 207,209, 309C] & 68.20 & 29.71 & $38.50(29.38,47.61)$ & $<0.001$ \\
\hline Confidentiality [Q205, 213] & 76.75 & 65.29 & $11.46(2.21,20.71)$ & 0.015 \\
\hline Participant right [Q202, 210, 212, 304, 305, 306] & 70.32 & 27.64 & $42.67(36.35,48.99)$ & $<0.001$ \\
\hline \multicolumn{5}{|l|}{ Summary comprehension score } \\
\hline aUnderstanding & 73.01 & 45.61 & $27.40(22.60,32.19)$ & $<0.001$ \\
\hline${ }^{b}$ Recall & 73.16 & 44.78 & $28.37(23.34,33.40)$ & $<0.001$ \\
\hline Over all C-score & 73.09 & 45.17 & $27.92(23.97,31.87)$ & $<0.001$ \\
\hline
\end{tabular}

anderstanding -score of Q201, 202, 205, 209, 210, 211, 212, 213, 301, 304, 305, 306, 307

${ }^{\mathrm{b}}$ Recall- score of Q 203, 204, 206, 207, 208, 302, 303, 308A, 308B, 309C, 310A, 310C, 311A, 312A, 312B

as the study was expected to generate ethical issues related to the sensitivity of the subject, ambiguity of terms, and decision-making dynamics of the community. The study was conducted in a well-defined population group with relative homogeneity in language, ethno-cultural and geographic parameters. The two study sites (intervention and control) were similar to each other on major societal parameters. They were geographically adjacent to each other and belonged to the same ethno-cultural cosmos including the health system. The socio-demographic characteristics of study participants in both locations revealed overall similarity except few irregularities. We employed MICCA and BIQ for measuring comprehension and QuIC for assessing quality of informed consent. The tools were able to capture both standard informed consent items as well as studyspecific test items [17]. Certain allowable practical adjustments were done on the tools and some of the components, otherwise both tools have been validated for use by researchers in different settings [17].

Recruitment rate and the recruitment index are considered important parameters in monitoring the quality of research implementation [6, 7]. In this study higher rates of both recruitment and retention were documented for the intervention group. While we documented strong statistical association between study retention rate and use of REA-based revision on informed consent process, no statistically significant difference was documented between the recruitment rates of the two groups. Previous studies have tried to identify potential contributors to recruitment rates of study participant such as 'opt-out' approaches [20], early consideration of participants' perspectives [21], addressing ethical issues such as the therapeutic misconception [6], and establishing trust between researchers and potential participants [22]. The recruitment rate, however, may have been affected by other factors beyond the consent information as the complexities of the challenges of recruitment are also determined by the complexities of the respective studies [7, 23, 24] and decisions being made prior to recruitment based on information circulating in the community [25].

Recruitment rate has been documented as an important determinant of subsequent retention [6, 24]. Other factors affecting retention include mental state of the person [26], availability of tracking and follow-up mechanisms [27], availability of mechanisms addressing community and context-specific issues and needs in special communities [28]. However every study has its own peculiar features and it may be difficult to generalise these factors. The registered improvement in the study retention rate in our study may have resulted from improved understanding of the consent information.

The current study documented significant differences in the comprehension levels (understanding and recall) between the intervention and control groups. REAbased adjustments to the content and delivery of consent information in the intervention group were linked to the improved levels of understanding and recall in the intervention group. These findings concur with studies which have reported improved consent information comprehension with appropriate interventions such as provision of more explanation and socio-culturally tailored 
Table 7 Variables associated with comprehension status among study participants in WukiroKilte-awulaelo and Hintalo-wajirat districts, Tigray region, Northern Ethiopia, July 2013

\begin{tabular}{|c|c|c|c|c|}
\hline \multirow[t]{2}{*}{ Variables } & \multicolumn{2}{|c|}{ Comprehension score status $(N=199)$} & \multirow[t]{2}{*}{ AOR $(95 \% \mathrm{Cl})$} & \multirow[t]{2}{*}{$P$-value } \\
\hline & High $(>=75 \%) f(\%)$ & Low $(<75 \%) f(\%)$ & & \\
\hline \multicolumn{5}{|l|}{ Age in years } \\
\hline $18-24$ yrs. & $23(34.3 \%)$ & 49 (37.1\%) & $1.09(0.38,3.10)$ & 0.876 \\
\hline $25-31$ yrs. & $26(38.8 \%)$ & 49 (37.1\%) & 1 ( Reference gp) & \\
\hline $32-38$ yrs. & $16(23.9 \%)$ & $28(21.1 \%)$ & $1.7(0.64,4.55)$ & 0.285 \\
\hline \multicolumn{5}{|l|}{ Marital status } \\
\hline Married & $58(86.6 \%)$ & $108(81.8 \%)$ & 1 (Reference gp) & \\
\hline Single & $3(4.5 \%)$ & $10(7.6 \%)$ & $1.05(0.17,6.58)$ & 0.955 \\
\hline Divorced & $4(6.0 \%)$ & $9(6.8 \%)$ & $0.59(0.14,2.53)$ & 0.479 \\
\hline \multicolumn{5}{|l|}{ Religion } \\
\hline Ortho. Christian & $54(80.6 \%)$ & $118(89.4 \%)$ & 1 (Reference gp) & \\
\hline Muslim & $13(19.4 \%)$ & $10(7.6 \%)$ & $2.4(0.80,7.15)$ & 0.089 \\
\hline \multicolumn{5}{|l|}{ Educational status } \\
\hline Not able to read and write & $13(19.4 \%)$ & $45(34.1 \%)$ & 1 (Reference gp) & \\
\hline Able to read and write & $6(9.0 \%)$ & $11(8.3 \%)$ & $2.20(0.48,10.15)$ & 0.314 \\
\hline Within or completed primary school & $15(22.4 \%)$ & $35(26.5 \%)$ & $2.23(0.57,8.72)$ & 0.251 \\
\hline Within or completed secondary school & $20(29.9 \%)$ & $31(23.5 \%)$ & $2.37(0.52,10.86)$ & 0.266 \\
\hline Diploma and above & $13(19.4 \%)$ & $10(7.6 \%)$ & $6.68(1.01,44.03)$ & 0.048 \\
\hline \multicolumn{5}{|l|}{ Occupation } \\
\hline House wife & $8(11.9 \%)$ & $63(47.7 \%)$ & 1 (Reference gp) & \\
\hline Private employee & $4(6.0 \%)$ & $10(7.6 \%)$ & $3.39(0.64,17.92$ & 0.151 \\
\hline Government employee & $15(22.4 \%)$ & $13(9.8 \%)$ & $2.91(0.62,13.67)$ & 0.177 \\
\hline Merchant & $18(26.9 \%)$ & $16(12.1 \%)$ & $5.43(1.78,16.60)$ & 0.003 \\
\hline Farmer & $1826.9 \%)$ & $24(8.2 \%)$ & $9.05(2.79,29.23)$ & 0.000 \\
\hline \multicolumn{5}{|l|}{ Monthly family income } \\
\hline$=<500 \mathrm{ETB}$ & $3(4.5 \%)$ & $13(9.8 \%)$ & $0.67(0.13,3.35)$ & 0.623 \\
\hline$>500 \&<1000 \mathrm{ETB}$ & $9(13.5 \%)$ & $51(38.6 \%)$ & $0.38(0.15,0.96)$ & 0.041 \\
\hline$>=1000$ ETB & $55(82.1 \%)$ & $68(51.5 \%)$ & 1 (Reference gp) & \\
\hline \multicolumn{5}{|l|}{ Previous participation } \\
\hline Yes & $21(18.4 \%)$ & $15(17.6 \%)$ & $2.77(1.01,7.58)$ & 0.047 \\
\hline No & $85(74.6 \%)$ & $60(70.6 \%)$ & 1 (Reference gp) & \\
\hline
\end{tabular}

approach [29]. Provision of tailored terminologies and additional narrative explanations on the pre-identified ethical issues and allowing more time for discussion and questions from the participants in the intervention group might have been potential determinants. There was no significant difference in comprehension levels related to disease information between the intervention and control group. The reasons for non-significance could be the sensitivity and novelty of the issues of cervical cancer in the area.

In the study there was a significant difference in the overall levels of perceived quality of the consent process in the intervention compared to the control group. There was a statistically significant difference in all but three of the components of quality assessment; whether consent form was read and explained carefully; whether consent from was important source of information; and whether consent form was important for consent decision. Possible reasons for the improved perception of the quality of the consent process in this study include the improvements made in the consent information and the revised consent procedures both of which were geared to the needs and concerns of the potential participants, based on the REA. There are several possible reasons for lack of statistically significant improvements in three areas. Regarding readability, the participants in the intervention groups mainly 
Table 8 Comparison of mean quality of informed consent process (QulC) score from the participant's perspective by intervention group in WukiroKilte-Awulaelo and Hintal-wajiratdistricts, Tigray region, Northern Ethiopia, July 2013

\begin{tabular}{|c|c|c|c|c|}
\hline \multirow[t]{2}{*}{ Informed consent process components } & \multicolumn{3}{|c|}{ Quality of IC (Mean \%) } & \multirow[b]{2}{*}{$P$ value } \\
\hline & $\begin{array}{l}\text { Intervention } \\
(n=114)\end{array}$ & $\begin{array}{l}\text { Control } \\
(n=85)\end{array}$ & $\begin{array}{l}\text { Mean difference } \\
(95 \% \mathrm{Cl})\end{array}$ & \\
\hline There was sufficient time for consent discussion. [Agree] & 93.9 & 82.4 & $11.5(2.7,20.3)$ & 0.010 \\
\hline Agreed to participate in this study voluntarily and with full understanding. [Agree] & 100 & 87.1 & $12.9(6.7,19.2)$ & $<0.001$ \\
\hline Enrolment decision made mainly by me the respondent. [Agree] & 99.1 & 92.9 & $6.2(1.0,11.3)$ & 0.019 \\
\hline Discussed about the research with other patients or participants. [Agree] & 91.2 & 17.6 & $8.9(0.5,18.2)$ & 0.062 \\
\hline Consent form read or explained carefully. [Agree] & 82.5 & 81.2 & $1.3(-9.7,12.2)$ & 0.818 \\
\hline Consent form was important source of information. [Agree] & 92.1 & 88.2 & $3.9(-4.5,12.2)$ & 0.361 \\
\hline Consent form was easy to understand. [Agree] & 97.4 & 90.6 & $6.8(0.4,13.2)$ & 0.039 \\
\hline Consent form was important to the decision. [Agree] & 95.6 & 95.3 & $0.3(-5.6,6.2)$ & 0.915 \\
\hline Pressure from provider to sign/agree consent form. [Disagree] & 95.6 & 76.5 & $19.1(10.1,28.2)$ & $<0.001$ \\
\hline Sufficient opportunity to ask questions. [Agree] & 95.6 & 67.1 & $28.6(18.8,38.3)$ & $<0.001$ \\
\hline Questions answered thoroughly by the consent provider. [Agree] & 99.1 & 74.1 & $25.0(16.6,33.4)$ & $<0.001$ \\
\hline Satisfied with informed consent process. [Agree] & 99.1 & 81.2 & $17.9(10.4,25.5)$ & $<0.001$ \\
\hline Decision to participate was easy or very easy. [Agree] & 99.1 & 87.1 & $12.1(5.5,18.6)$ & $<0.001$ \\
\hline Mean score of Quality of informed consent & 89.06 & 78.53 & $10.5(6.8,14.2)$ & $<0.001$ \\
\hline
\end{tabular}

had the form read to them rather than reading it themselves. Another reason is that all three measures already scored highly in the control group with little room for improvements. Though not significant, there were improvements observed in the three categories and if a larger sample had been used this might have been significant. Quality of informed consent depends on factors such as type of consent information provided, amount of information, adequacy of comprehension, and the voluntariness of decision making by the participants [5]. In the clinical set up, participant-related factors such as age, IQ, level of cognitive function and external locus of control were associated with poor quality and information recall. Written information provided immediately before admission was associated with better outcomes [30].

Various researchers have utilized different interventions to improve the consent process and understanding, such as use of local narratives [31], extended one-to-one discussion [32-34], enhanced consent [33, 35], a revised consent process [36], and a booklet of participants' rights [37]. Cultural and linguistic modifications to the informed consent process were considered to significantly enhance understanding by study participant in low income settings [29]. On the other hand it has been shown that lexicosyntactic readability improvement of the consent form [38], multimedia enhanced consent [34], and use of a concise version of the consent form [39] do not have effects on comprehension. There are still ongoing evidence-based debates as to which interventions are effective in enhancing comprehension [40]. The intervention in this study involved improvements changes in the consent procedures based on REA findings.

One of the ethical dilemmas faced by the researchers while revising the consent form is what level of change on an IRB-approved form would be appropriate and allowable once in the field, and what principles should govern this. According to existing guidelines, any amendments of an approved research project require further IRB approval before implementation $[41,42]$. The only exceptions to this are if the changes are immediately and urgently required for the safety of the subjects. In this case, the IRB must be informed immediately after establishing the need for the changes -in most cases within five business days [43]. The other possible exception to this rule is if the study is a determined to be non-research by the IRB and does not need further IRB follow-up [41]. We made revisions to the consent form in the field on the following basis; a) that the revision added to the approved consent and did not reduce any major component (the consent form content and structure approved by IRB were maintained with improvements); b) that the comprehension assessment project and the possibility of revising the consent form were already approved by IRB; c) that the changes were important for the welfare of the subjects in the study. Since getting approval from IRB for the modification might take time, some provision for making appropriate adjustment without tampering with the basic components of consent may be necessary in future.

The Ottawa Statement provides a framework for addressing ethical issues around cluster randomised trials. 
According to the statement on the Ethical Design and Conduct of Cluster Randomized Trials, "When participants' informed consent is required, but recruitment of participants is not possible before randomization of clusters, researchers must seek participants' consent for trial enrollment as soon as possible after cluster randomization-that is, as soon as the potential participant has been identified, but before the participant has undergone any study interventions or data collection procedures" $[44,45]$. In the current study consent was obtained soon after recruitment in to the planned study without further additional approval of the REA based revisions on the originally approved consent form, based on the premises presented above.

The study has some potential limitations. Efforts were made to ensure the uniformity of the intervention and control sites, however there may have been undocumented differences in the settings which may have confounded the study outcomes. Both levels of comprehension and quality of the informed consent process are based on participant perceptions using questionnaire based interviews which were not accompanied by independent observation methods. This might reduce the objectivity and validity of the responses. Reasons for loss to follow-up of those who did not attend were not documented, and neither did we document the basic socio-demographic characteristics of non-attendees. The sample size determination was done based on assumptions from other countries as there was no local estimate available. In addition we assumed a $10 \%$ non-response rate while the non-response rate for the study was about $15 \%$. The single cluster intervention also might have reduced the study power.

\section{Conclusions}

Based on our findings, REA is associated with improved understanding of study concepts, purposes and procedures and participants' perceptions of the informed consent process. Investing in an intervention which enhances researchers' understanding of the local context and tailoring the consent information and process is associated with improvement $\mathrm{s}$ in overall consent comprehension, study recruitment rates and retention rates in low-income settings. REA is also an appropriate intervention for further use in similar settings and situations.

We would like to encourage researchers and research ethics committees and policy makers to employ REA to improve research consent comprehension, recruitment and retention levels in certain low income settings. As this is the first study exploring the effects of REA on comprehension, additional studies in other settings with more objective methods of measurement would provide greater insight into the overall effects of REA on comprehension of consent. Such studies might even expand into consent processes for medical interventions. Ethicists and research policy makers are encouraged to explore the consequences of utilizing REA in the light of IRB requirements and operating procedures. In future studies, if REA is planned, it should be included in the research proposal, so it and any subsequent consent form modification are approved by the IRB.

\section{Additional files}

Additional file 1: Standard information sheet and Consent form for "HPV-Subtype Prevalence Study". This version is the non-modified information sheet and consent form, the version initially approved by the IRB. This is the very version of information sheet and consent form administered used the control group. (DOCX 17 kb)

Additional file 2: Modified information sheet and consent form for "HPV-Subtype Prevalence Study". This is the modified version of information sheet and consent form administered for the intervention group. Modifications were done on the IRB approved version based on the REA (Rapid Ethical Assessment) conducted prior recruitment. (DOCX 21 kb)

Additional file 3: Data collection materials used for comprehension assessment in "HPV-subtypes Prevalence Study". These are the data collection material used for testing comprehension and quality of informed consent in both the control and intervention groups. The material were developed based on Modular Informed Consent Comprehension Assessment (MICCA), Brief Investigator Questionnaire (BIQ) and Quality of Informed Consent (QulC) tools. (DOCX 28 kb)

\section{Abbreviations}

ANC, Ante natal care; BIQ, Brief investigator questionnaire; HPV, Human papilloma virus; IRB, Institutional review board; MICCA, Modular informed consent comprehension assessment; QuIC, Quality of informed consent; REA, Rapid ethical assessment; SPSS, Statistical package for social sciences

\section{Acknowledgements}

We would like to thank all the research institutions involved in the study. We also would like to thank all the individuals participating in the interviews and on-line survey. We are very thankful to Wellcome Trust for the financial support.

\section{Funding}

The study was financially supported by Wellcome Trust (WT) through Biomedical Ethics Doctoral Fellowship 089769. However WT has no any part in the execution of the study and write-up.

\section{Availability of data and materials}

Public availability would compromise patient privacy. Data will be made available on request from The Wellcome Trust Brighton and Sussex Center for Global Health Research. Requests for access to anonymised data should be made via Debbie Miller (d.miller@bsms.ac.uk)'.

\section{Authors' contribution}

$A A, S A, G D$, and BF designed the study. MN and YF critically evaluated and made progressive suggestions on the initial study design. AA and SA developed and refined study instruments. SA led field data collection and analysis. All authors (AA, SA, GD, BF, MN, and YF) were involved in the write up of the manuscript and in the critical review of drafts. All authors read and approved the manuscript.

\section{Competing interests}

The authors declare that they have no competing interests (financial and non-financial).

\section{Ethical approval and consent to participate}

The research project was approved by the Institutional Review Board of the College of Health Sciences, Addis Ababa University in Ethiopia and the Research Governance and Ethics Committee at Brighton and Sussex Medical School in the UK. Consent was obtained from all study participants after providing adequate information about the research. 


\section{Author details}

${ }^{1}$ Brighton and Sussex Medical School, Brighton, UK. ${ }^{2}$ Addis Ababa University, Addis Ababa, Ethiopia. ${ }^{3}$ Ethiopian Public Health Institute, Addis Ababa, Ethiopia.

Received: 13 December 2014 Accepted: 30 June 2016

Published online: 12 July 2016

\section{References}

1. Sanchez $\mathrm{S}$ et al. Informed consent procedures: responsibilities of researchers in developing countries. Bioethics. 2001;15(5-6):398-412.

2. Sugarmann J et al. Evaluatng the Quality of Informed Consent. Clin Trials. 2005;2:34-41.

3. Minnies $D$ et al. Evaluation of the quality of informed consent in a vaccine field trial in a developing country setting. BMC Med Ethics. 2008;9:15.

4. Kiguba $\mathrm{R}$ et al. Assessing the quality of informed consent in a resourcelimited setting: A cross-sectional study. BMC Med Ethics. 2012;13:21.

5. Mandava A et al. The quality of informed consent: mapping the landscape A review of empirical data from developing and developed countries. J Med Ethics. 2012;38(6):356-65.

6. Blanton S et al. Lessons Learned in Participant Recruitment and Retention The EXCITE. Trial Phys Ther. 2006:86:1520-33.

7. Galea S, Tracy M. Participation Rates in Epidemiologic Studies. Ann Epidemiol. 2007:17:643-53.

8. Hill Z et al. Informed Consent in Ghana: what do participants really understand? J Med Ethics. 2008;34:48-53.

9. Woodsong C, Karim QA. A model designed to enhance informed consent: experiences from the HIV prevention trials network. Am J Public Health. 2005;95(3):412-9.

10. Marshall PA. "Cultural competence" and informed consent in international health research. Camb Q Healthc Ethics. 2008;17(2):206-15.

11. Bull SJ. Consent to research in a Gambian context: legal, social and ethical issues (PhD thesis). London: Kings College; 2007.

12. Tekola F et al. Tailoring consent to context: designing an appropriate consent process for a biomedical study in a low income setting. PLoS Negl Trop Dis. 2009;3(7):e482.

13. Bull S, Farsides B, Tekola Ayele F. Tailoring information provision and consent processes to research contexts: the value of rapid assessments. J Empir Res Hum Res Ethics. 2012;7(1):37-52.

14. Tindana $P$ et al. Seeking consent to genetic and genomic research in a rural Ghanaian setting: A qualitative study of the MalariaGEN experience. BMC Med Ethics. 2012;13(15):1-12.

15. Addissie A et al. A mixed-methods study on perceptions towards use of Rapid Ethical Assessment to improve informed consent processes for health research in a low-income setting. BMC Med Ethics. 2014;15(35):1-22.

16. Assefa M. HPV in women of Ethiopia: an approach to cervical cancer (Research Project Proposal). Addis Ababa, Ethiopia: Addis Ababa University; 2012.

17. Buccini L. Developing an instrument to measure informed consent comprehension in non-cognitively impaired adults, in School of Health Sciences. Wollongong: University of Wollongong; 2009a.

18. Joffe $S$ et al. Quality of Informed Consent: a New Measure of Understanding Among Research Subjects. J Natl Cancer Inst. 2001;93:139-47.

19. Buccini $L$ et al. Toward a construct definition of informed consent comprehension. J Empir Res Hum Res Ethics. 2009:4(1):17-23.

20. Junghans $C$ et al. Recruiting patients to medical research: double blind randomised trial of "opt-in" versus "opt-out" strategies. BMJ. 2005;331:1940-42.

21. Patel M, Doku V, Tennakoon L. Challenges in recruitment of research participants. Adv Psychiatr Treat. 2003;9:229-38.

22. Kneipp S, Lutz B, Means D. Reasons for Enrollment, the Informed Consent Process, and Trust Among Low-Income Women Participating in a Community-Based Participatory Research Study. Public Health Nursing 2009;26(4):362-9.

23. McDonald A et al. What influences recruitment to randomised controlled trials? A review of trials funded by two UK funding agencies. Trials. 2006;7(9):1-8.

24. Frank G. Current Challenges in Clinical Trial Patient Recruitment and Enrollment SoCRA SOURCE. 2004:30-38.

25. Paré Toe $L$ et al. Could the Decision of Trial Participation Precede the Informed Consent Process? Evidence From Burkina Faso. PLoS ONE. 2013;8(11):e80800.
26. Chang M, Brown R, Nitzke S. Participant recruitment and retention in a pilot program to prevent weight gain in low-income overweight and obese mothers. BMC Public Health. 2009;9:424.

27. Murugesan, A., et al. Achieving High Retention Rate of Clinical Trial Subjects in A Long Term Study Among Rural Population. in 69th AIOC. Ahmedabad: Addis Ababa University; 2011.

28. Ejiogu N et al. Recruitment and Retention Strategies for Minority or Poor Clinical Research Participants: Lessons From the Healthy Aging in Neighborhoods of Diversity Across the Life Span Study. Gerontologist. 2011;51(S1):S33-45.

29. Penn C, Evans M. Assessing the impact of a modified informed consent process in a South African HIV/AIDS research trial. Patient Educ Couns. 2010;80:191-9.

30. Lavelle-Jones $\mathrm{C}$ et al. Factors affecting quality of informed consent. BMJ. 1993:306:885-90.

31. Ndebele P et al. Improving understanding of clinical trial procedures among low literacy populations: an intervention within a microbicide trial in Malawi. BMC Med Ethics. 2012;13(29):1-14.

32. Tamariz L et al. Improving the Informed Consent Process for Research Subjects with Low Literacy: A Systematic Review. J Gen Intern Med. 2012;28(1):121-6.

33. Nishimura A et al. Improving understanding in the research informed consent process: a systematic review of 54 interventions tested in randomized control trials. BMC Med Ethics. 2013;14(28):1-15.

34. Flory J, Emanuel E. Interventions to Improve Research Participants Understanding in Informed Consent for Research. A Systematic Review. JAMA. 2004:292:1593-601.

35. Dunn $L$ et al. Enhancing Comprehension of Consent for Research in Older Patients With Psychosis: A Randomized Study of a Novel Consent Procedure. Am J Psychiatry. 2001;158:1911-3.

36. Sudore R et al. Use of a Modified Informed Consent Process among Vulnerable Patients. A Descriptive Study. J Gen Intern Med. 2006;21:867-73.

37. Benatar J et al. A Booklet on Participants' Rights to Improve Consent for Clinical Research: A Randomized Trial. PLoS ONE. 2010;7(10):e47023.

38. Paris A et al. Informed consent document improvement does not increase patients' comprehension in biomedical research. Br J Clin Pharmacol. 2009;69(3):231-7.

39. Enama $\mathrm{M}$ et al. Randomization to Standard and Concise Informed Consent Forms: Development of Evidence-Based Consent Practices. Contemp Clin Trials. 2012:33(5):895-902.

40. Cohn E, Larson E. Improving Participant Comprehension in the Informed Consent Process. J Nurs Scholarsh. 2007;39(3):273-80.

41. Johns Hopkins Medicine. Frequently Asked Questions. 2013; Available from: http://www.hopkinsmedicine.org/institutional_review_board/about/faqs. html. Accessed 10 July 2016.

42. University of Northern Texas Health Science Center. Amendments and Modifications to a Research Project. 2015; Available from: https://www. unthsc.edu/research/protection-of-human-subjects/protocol-amendments/. Accessed 10 July 2016

43. University of Connecticut Health Center. Questions Related to the Need for IRB Review. 2013. Available from: http://hspo.uchc.edu/faq/index.html. Accessed 10 July 2016

44. Weijer $C$ et al. Ethical issues posed by cluster randomized trials in health research. Trials. 2011;12:100.

45. Weijer $C$ et al. The Ottawa Statement on the Ethical Design and Conduct of Cluster Randomized Trials. PLoS Med. 2012;9(11):e1001346.

\section{Submit your next manuscript to BioMed Central and we will help you at every step:}

- We accept pre-submission inquiries

- Our selector tool helps you to find the most relevant journal

- We provide round the clock customer support

- Convenient online submission

- Thorough peer review

- Inclusion in PubMed and all major indexing services

- Maximum visibility for your research

Submit your manuscript at www.biomedcentral.com/submit 